\title{
Parque Ribalta de Castellón
}

\author{
Encarna Ballester Mir
}

\section{Antecedentes}

El parque Ribalta está situado al noroeste de la ciudad de Castellón. Hasta el año 1860 fue el cementerio de la ciudad llamado de El Calvario, que se construyó en 1804 en su entonces lejano emplazamiento de la ciudad, en El Arrabalet, a 400 metros de las últimas casas y a 950 metros de la iglesia Mayor, fuera da las murallas de la ciudad por el portal de La Fira, que más tarde se llamaría el Portal de Morella, derribado en 1884, y más allá de la ermita del Calvario, de ahí su nombre. Al expandirse la ciudad hacia el oeste otra vez, quedó demasiado cerca de la ciudad y tuvo que ser derribado y hubo de construirse otro hacia el oeste y en la misma linea, al otro lado del río Seco, con más cabida.

\section{Primer parque}

En 1868, el 12 de noviembre, siendo alcalde D. Carlos Ferrer, el Ayuntamiento acordó construir en el solar del antiguo cementerio de El Calvario, de una viña de D. Salvador Tárrega, de un secano de D. José de la Fuente y otro del conde de Pestagua, un parque. Sus dimensiones, en un principio, eran de 9 hanegadas y 3 cuartones. El 19 de noviembre se aprueba el proyecto de D. Luis Alfonso y Brull que se ofreció además a dirigir los trabajos y cedió sus honorarios al Ayuntamiento para subsanar las obras.

El mismo día que se presentó el proyecto, se acordó darle el nombre de paseo Ribalta, ya que se creía entonces que había nacido en Castellón, aunque, tras los estudios realizados últimamente, se sabe que nació en Solsona (Lérida) en 1565 y falleció en Valencia en 1628. Aún así, en Castellón lo siguen considerando hijo de la ciudad.

Los trabajos de jardinería se deben al funcionario de jardines del Ayuntamiento D. Tomás Clará Ferrer, quien aprovechó todo el arbolado que pudo del antiguo cementerio (los cipreses del camino) plantó álamos y unos pinos traídos del pinar del Grao.

\section{Ampliación}

En el año 1876, siendo alcalde D. Domingo Herrero, se acordó en el pleno municipal construir, junto al entonces paseo Ribalta, el paseo del Obelisco, llamado en su día de la Alameda (pleno del

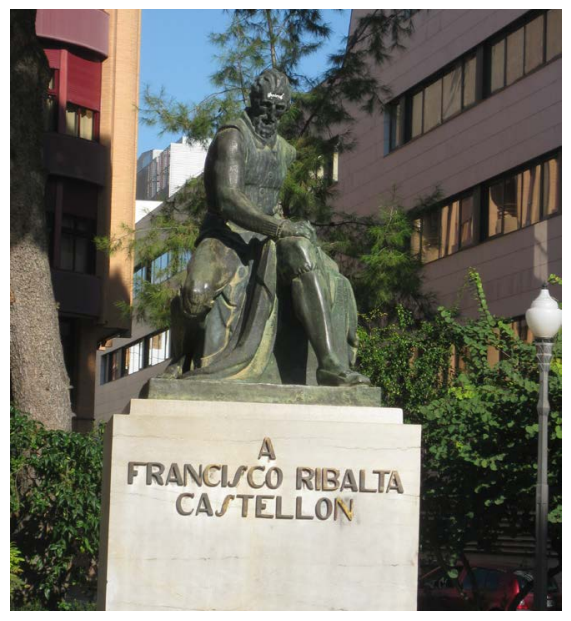

Figura 1. Estatua a Francisco Ribalta 
10 de febrero de 1877) y que el pueblo le llamaba el paseo nuevo.

Para realizar el proyecto, era necesario expropiar unos terrenos valorados en 2801 pesetas. Estos eran: una viña, un olivar, dos algarroberos y dos campos de secano. Después de mucho tratar, se llegó a un acuerdo con el conde de Pestagua en el que este cedía gratuitamente los terrenos a cambio de quedarse un algarrobal en toda la parte norte del parque.

En la esquina noroeste, la viuda de Miguel Tomás tenía una casa y un terreno, como se puede ver en los planos de 1890, que se resistió a vender por 250 reales, por lo que Luis Alfonso tuvo que rectificar el proyecto primitivo. No se tienen datos de cuándo se llegó a un acuerdo, pero sí se sabe que casi 25 años después se llevó a cabo el acuerdo con la viuda del conde de Pestagua porque en los planos de primeros del s. $x x$ ya figura completo el parque.

D. Godolfredo Ros de Ursinos, el 9 de junio de 1877, sacó la plaza de arquitecto municipal de ahí su vinculación con el paseo. Este arquitecto tiene, en Castellón, otros edificios diseñados por él, como son la iglesia de la Sagrada Familia, el teatro Principal y otros, pero en el parque Ribalta solo intervino firmando algún documento, como arquitecto municipal, pero no diseñó el parque, como mucha gente cree; el verdadero artífice del parque fue $\mathrm{D}$. Luis Alfónso Brull.

El diseño es muy original, lleno de plazoletas con bancos de cerámica a los que se llega por sinuosos pasillos, componiendo todo él un complejo de la-

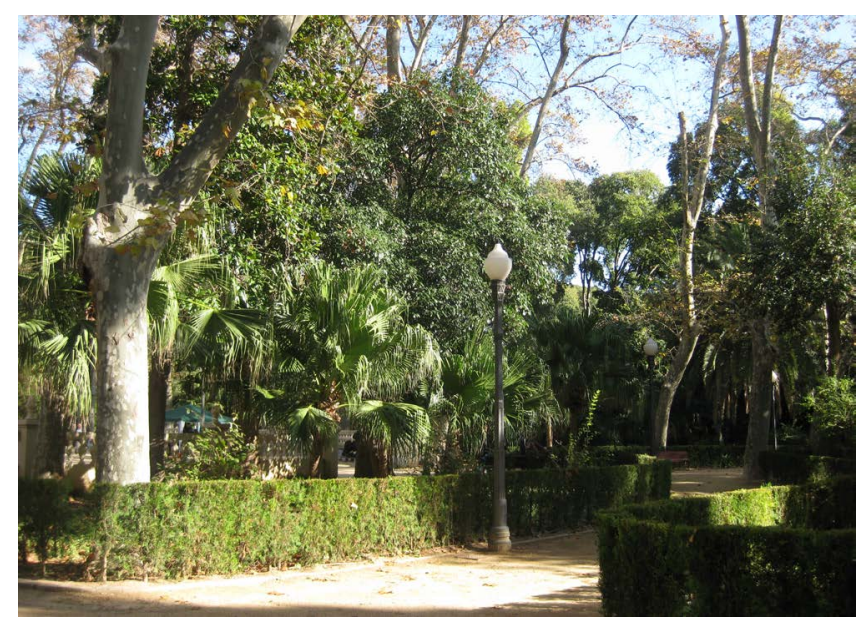

Figura 2. Detalle del interior del parque berintos que lo hacen muy peculiar y romántico, y lo cruzan unos paseos más amplios a modo de un tablero del juego tres en raya.

Al floricultor Sr. Tirado se le encomendó la tarea de planificar la vegetación de todo el parque. En un principio, la vegetación estuvo compuesta por pinos que se trasplantaron del pinar, eucaliptos y los cipreses que quedaron del antiguo cementerio. Más tarde se plantaron álamos, plataneros, tuyas, palmáceas, celtis o lledoners y cipressus funebrius.

No seria hasta diez años después cuando se plantea dotar de vegetación al paseo del Obelisco y unificar todo el parque, y es entonces cuando se plantan, con el pensar y estilo de la época, árboles exóticos y plantas singulares traídas de muy diferentes partes del mundo o aclimatadas ya en jardines botánicos. De esta época es también el jardín junto a la estación de Nules y el jardín botánico y la glorieta de Valencia, que son de las mismas características.

Se tiene constancia de que, en 1897, existían al menos dos viveros que abastecían de plantas al parque y que uno estaba por cerca de Lidón y el otro en el pinar del Grao. En documentos datados en 1902, se dice que en el parque hay aligustres, plátanos, tuyas y eucaliptus.

Hay que decir que estos dos paseos lindaban por el oeste con la vía del ferrocarril de Almazora-Valencia-Tarragona, ferrocarril que llegó a Castellón en 1862 con la Compañía Norte. La estación se construyó en el lado suroeste del parque. Hoy en día es lo único que queda en recuerdo de su paso junto al parque es la estación, porque, como ha seguido creciendo la ciudad hacia el oeste en los últimos tiempos, el ferrocarril se ha trasladado más hacia el oeste y se han soterrado las vías.

\section{Mejoras}

En el año 1882 se abre la muralla, por donde se encuentra hoy la calle San Vicente, para comunicar mejor la estación del ferrocarril con la ciudad, quedando así limitado el parque por el sur con el camino de la Estación, y se plantaron, en todo su largo, una hilera de plataneros, dando así más vida al parque. 
El portal de Morella se derribó en 1984 para facilitar mejor el acceso a la nueva carretera de Castellón a Zaragoza, pasando por Morella, construida el año 1860. Hasta entonces, a Zaragoza se pasaba por Teruel y se accedía a ella por la carretera de l'Alcora.

La iluminación de gas del parque se inauguró en agosto de 1886.

En 1902 se construyó la caseta del motor del pozo de ladrillo con juegos mudéjares; dirigió las obras el arquitecto municipal D. Godolfredo Ros de Ursinos, por lo que se cree que la diseño él mismo.

Entre los dos parques, Ribalta y Obelisco, pasaba la carretera de Castellón-Zaragoza por Morella, pero como esto no gustaba, en 1920 se desvió por el lado norte del primitivo paseo Ribalta, quedando así unidos los dos parques, unificándose y llamándose desde entonces a todo el parque Ribalta.

En 1898, en el paseo del Obelisco, antes de la Alameda, se erigió un obelisco en piedra labrada de Borriol sobre un pedestal cuadrado y rodeado de verja de hierro octogonal, en conmemoración del sitio de Castellón, los días 7, 8 y 9 de julio de 1837 por las tropas carlistas dirigidas por el general Cabrera, que, aunque sitió y quiso entrar a Castellón por el Arrabalet (donde estaba el cementerio del Calvario), gracias al valor de los castellonenses no logró entrar y siguió hacia Vila-real. Este acto fue reconocido por la reina Isabel II que alabó el valor de las gentes de Castellón y la llenó de honores. Erigió a la villa en ciudad y le dio el titulo de «fiel y leal». Más tarde, por los servicios prestados a la patria en 1876, le añadió los títulos de «constante y de excelente».

En 1939 fue demolido y lo reconstruyó D. Tomás Traver en 1982, añadiendo en dos de las caras leyendas con fragmentos de textos de la Constitución y en las otras dos leyendas relatadas con el hecho tan glorioso del sitio de Castellón.

El paseo del Obelisco es el paseo preferido de los castellonenses por estar en el centro del paseo. Esta rodeado de un banco circular que invita a sentarse y la diagonal. Es por eso por lo que en 1899 se instala en la parte oeste del círculo el primer kiosco de venta de helados y refrescos; es el tradicional kiosco Campos, que duró hasta 1991. El señor Arruzat,

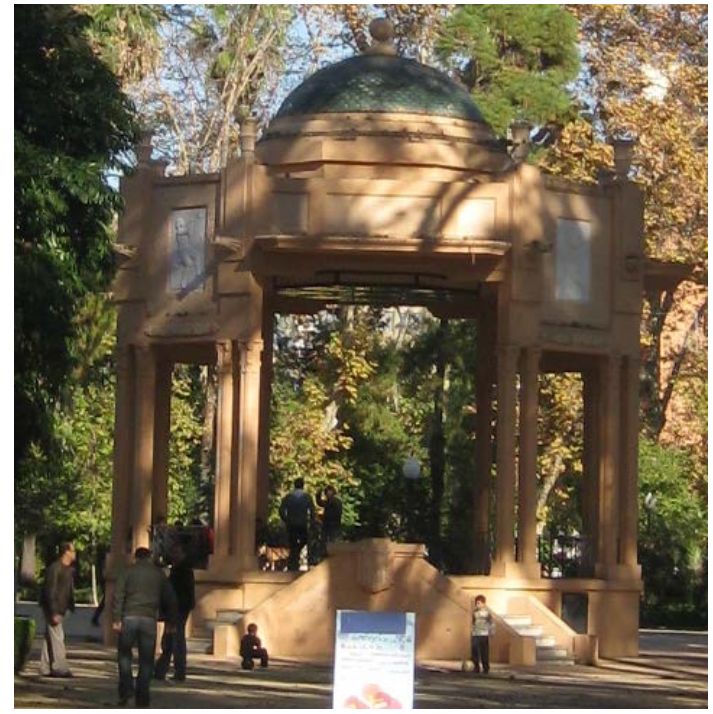

Figura 3. Templete

en 1915, solicitó otro kiosco que le fue permitido en 1917 y hay constancia de la solicitud de otro por José Martí y otro por Ramón Pinto Vilar y otro más junto al estanque en 1921. Existen indicios de que hubo otro de Vicente Ros Gimeno y otro del S. Mestre. Esto afirma que en esos años el paseo del Obelisco era verdaderamente el centro de reunión de la ciudad.

Se continuaron las mejoras y en 1903 se construyó un pozo en el paseo del Obelisco, pudiéndose así colocar fuentes públicas en el paseo y en la ciudad y en 1913 se construyeron mingitorios y se cambiaron las farolas de gas que se habían instalado el 16 de agosto de 1886 por las de luz eléctrica.

Cuando por fin se llegó a un acuerdo con la viuda del conde de Pestagua y el Ayuntamiento consiguió el campo de algarrobos como se ha comentado antes, se proyectó la construcción de un estanque el 24 de julio de 1914, obra del arquitecto municipal D. Francisco Tomás Traver, que tenía una doble finalidad: el embellecer la zona y disponer de la suficiente agua para el riego. Se presupuestó con 13118,25 pts. incluido en él el kiosco de los patos. Mide $1870 \mathrm{~m}$ cúbicos, suficiente para regar las 3 hectáreas y 74 áreas del terreno del parque.

El estanque tiene formas sinuosas que le dan un aire romántico que se acrecienta con los cisnes, patos, gansos y peces que lo habitan. Lo adornaba también una pérgola de madera del mismo estilo romántico y la estatua de una Venus que surge 


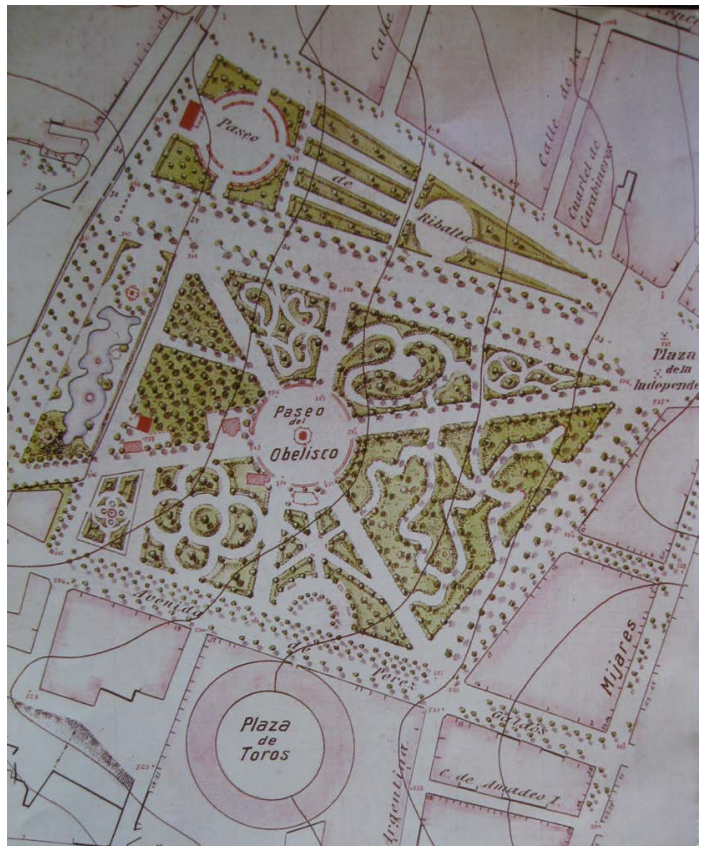

Figura 4. Plano del parque

elegantemente de las aguas, regalo de Valencia y a la que unos incívicos le arrancaron la cabeza. En 1996 se erigió otra de D. José Ruiz Correa que es la que existe actualmente.

En 1920, al trasladarse la carretera de Castellón a Zaragoza a la parte norte del parque, se construyó el salón de coches o de fiestas entre los dos parques, uniéndose así los dos parques que, aunque formaban un conjunto, los dividía la carretera. Este salón, al igual que los Campos Elíseos de París o la Alameda y la Glorieta de Valencia, era el centro social de su tiempo.

Las ferias que se celebraban en el Arrabalet alrededor del Calvario, fuera de las murallas desde la antigüedad, volvieron a celebrarse, desde 1916, en este salón de coches o de ferias. Las únicas ferias que se celebran eran la de la Magdalena y de Todos los Santos y se siguieron celebrando en este lugar hasta 1991.

En 1922 se compra una regadora automática que costó 39500 ptas.

El año 1932, D. Francisco Maristany Casajuana construyó el templete de la música; en 1966 se sustituyo el cielo raso por forjados y falso techo y en el 2002 se restauró por completo. Está dedicado a los músicos Chapí, Falla, Bretón y Giner y en él se celebran conciertos todos los domingos por la mañana hasta la actualidad. Es de estilo modernista y hay cuatro bajorrelieves alegóricos a los músicos. Está considerado como suntuoso y de gran belleza artística y decorativa. Costó 19821 pts.

En el primitivo parque infantil se levantó la cruz de los caídos. Se mandó construir el 30 de marzo de 1943 y se celebró el primer acto el 29 de marzo de 1944. Hoy, remodelada y sustituida la fuente por una rosaleda de rasas amarillas, está dedicada a las víctimas de la violencia. Se ubica en la parte este del parque, por donde se une con la plaza de Huerto de Sogueros, por una avenida de 30 metros adornada con bancos y palmeras.

En la parte sur del parque, frente a la plaza de toros, primero existió una biblioteca, que más tarde fue sustituida en el año 1936 por escuelas públicas, y en el año 1966 se construyó en este lugar un parque infantil de tráfico. Cuando este se derribó por quedar pequeño y anticuado para este fin se transformó en jardín llamado de los círculos.

Hoy, en esta parte sur frente a las puertas de la plaza de toros, se ha construido, en el año 2003, una media rotonda y erigido una estatua dedicada los críticos taurinos.

\section{Actualidad}

Siguiendo en esta zona sur, más hacia el oeste, estaba situado un parque infantil de juegos desde el año 1944 que se construyó la cruz de los caídos, y que en el año 1992 volvió a ser otra vez la rosaleda tal como se ve en el plano atribuido a Juan Mañá. Los primeros datos de su existencia los conocemos por facturas del año 1923 y se construyó tal como había sido diseñada en un principio siguiendo los planos primitivos de D. Juan Maña.

Los juegos infantiles desde 1992 se encuentran en la plaza del Obelisco.

Desde el año 2007, en la esquina suroeste, frente a la antigua estación, hay una monumental estatua dedicada al toro bravo de Melchor Zapata.

En 1941, en el lugar de los antiguos mingitorios, al ser destruidos estos por un huracán, se construyó un precioso palomar que aún existe en la actualidad y, muy cerca de él, la caseta del pou, construida en 1904 y mejorada actualmente. 
En el primitivo paseo Ribalta existía desde casi sus comienzos la pérgola, situada junto a unas casetas que las utilizaban los peones de labranza, rodeadas por un banco de sillería y un pequeño estanque junto a la fuente del Piulet.

La pérgola, cuando por las nuevas costumbres decayó el salón de coches, pasó a ser el centro de reunión donde se desarrollaba la vida social de Castellón hasta que se abandonó su uso por los cambios de gustos de la sociedad y, en su lugar, en 1971, se construyó un edificio circular cubierto que ha sido y aún es este año (aunque en 2011 se aprobó su derribo) testigo de todos los acontecimientos sociales de la ciudad y al que se le dio también el nombre de la pérgola. El nuevo edificio de eventos sociales constuido en Pau Taxida podría, siguiendo la tradición, llamarse también la nueva pérgola.

Estos últimos años se ha mejorado y se sigue mejorando el parque, sobre todo desde que en 1981 fue declarado conjunto histórico artístico. Está adornado con muchísimas estatuas y la principal y con la que estaba en deuda la ciudad de Castellón es la del pintor Francisco Ribalta, obra del escultor Juan Adsuara Ramos y que, muchos años después de decidir construirla, no se realizó hasta 1926. Es una de las grandes obras del escultor y posee un equilibrio de formas, una cuidada anatomía que lleva trazos de abstracción sin llegar a la geometría y se encuentra situada en el centro del primitivo paseo Ribalta, rodeada de bancos de sillar como todos los que hay en el paseo desde la farola hasta la pérgola y que son de gran calidad artística y han sido restaurados últimamente.

Otras estatuas existentes son la de D. Domingo Herrero, junto al estanque; la de D. Francisco Tárrega, del escultor Adsuara; la de D. Vicente Castell pintor, obra de Octavio Vicent; la del Día Mundial del Niño, Unicef en 1971; la del Lleuero en 1991 en la entrada noreste del parque; la de D. Pere Mechain astrónomo en 2010 a pocos metros de la anterior; la del toro bravo de Melchor Zapata, la del reporteron taurino. Aunque el sentir de los castellonenses es que todavía no están todas las estatuas que deberían al ser este parque el lugar idóneo para ensalzar a personajes y hechos ilustres de la ciudad.

En el paseo del Obelisco, a la altura del estanque, se ubicó durante un tiempo una locomotora de tren

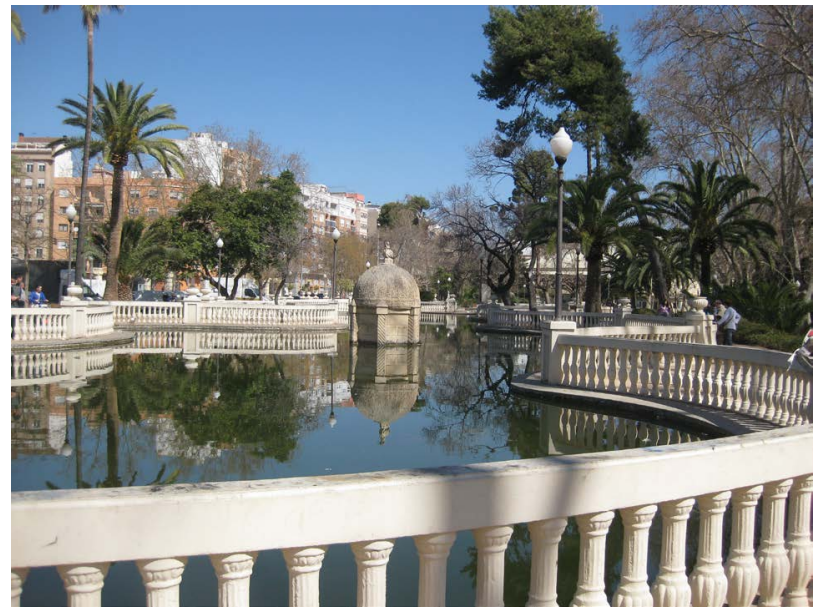

Figura 5. Estanque del parque

de vapor de vía estrecha que desde el 13 de agosto de 1880 y años sucesivos enlazaba el Grao con Castellón, Onda, Almazora, Vila-real, Burriana y Grao y al que cariñosamente los castellonenses llamaron la Panderola. Por la erosión, paso del tiempo y el maltrato de personas incívicas, se estropeó y llevó a reparar. Hoy en día sigue sin estar en el paseo en contra de lo que dice la canción: «i ara com ja no vola, xim pum tracatrac, està al paseo leré, leré». Una pena, porque gustaba de ver y recordaba años de juventud a los mayores que hoy disfrutan del paseo Ribalta.

En la actualidad, el parque está muy dinamizado y vuelve a ser centro de reunión y de esparcimiento tanto para mayores como para niños y jóvenes. Se realizan un gran número de actividades culturales y de recreo como conferencias, exposiciones, teatro, rutas guiadas, lectura, deporte, conciertos, etc. Sobre todo se ha convertido en espacio de recreo, paseo y relajación y un lugar idóneo para realizar ejercicio físico. A este fin se ha dispuesto de aparatos gimnásticos en en el espacio comprendido entre la verja norte y el estanque.

El paseo o salón de coches actualmente ya está remodelado y acondicionado para que en su día pase el tranvía eléctrico llamado el tram.

Entre las mejoras de estos últimos años se ha de destacar el gran cuidado que se tiene con la vegetación; todo el parque está al cuidado del arquitecto técnico D. Ramón Bachero y el arquitecto agrícola D. Jesús Albuixech Moliner y gestionado en la escuela Futura Metrópolis del excelentísimo Ayunta- 
miento de Castellón. Son los artífices de la nueva rosaleda.

El parque Ribalta hoy en dia mide 77787,5 metros cuadrados y en él hay plantados 2100 ejemplares de árboles, todos ellos catalogados con sus nombres científicos, su sinonimia y la región y el país de origen, por lo que, además de todas las funciones ya dichas, tiene la de ser el pulmón verde de la ciudad, de carácter educativo y de enseñanza. Todas las especies tienen su tarjeta de identificación. Se ha aireado el albolado, podándolo y modelándolo, se replantan las plantas, se pulverizan y se riegan cuanto hace falta, según la especie, y para ello se dispone de una red de riego con 3203 metros de cañeria y 116 bocas de riego que cubren todas las necesidades, que son muchas por la cantidad de vegetación que existe: 528 árboles, 5 585,5 metros de seto ligustrun japonicum y 784 de seto spyraea, además de rosales de distintas variedades, arbustos diversos y plantas anuales.

Ejemplo de arboles: casuarina, cólulo Kentia, pitosporo del Japón, árbol botella, yuca pie de elefante, árbol del amor, laurel, cerezo, malvaceas, yuca, almez, palmera canaria, pitosporum, y olmo como más significativas.

Es un gozo pasear por el parque: se respira paz y tranquilidad, da igual la hora o la estación del año, se oyen los pájaros, se huele la vegetación y las flores, se ven los distintos colores según la estación, se ven paseantes, señores jugando a la petanca, niños jugando, jóvenes haciendo ejercicio o, simplemente, personas dialogando.

Es un parque espléndido, extraordinario, maravilloso, el orgullo de Castellón.

\section{Referencias}

Balbas Cruz, J. A. (1892). El libro de la provincia de Castellón. Castellón.

Michavilla Gimeno, Vicente (1926). Del Castellón Viejo. Est. Tip. Hijo de J. Armengot. Castellón.

Mundina Milallave, B. (1873). Castellón. Historia, geografia y estadística. Rovira Hermanos. Castellón.

Santamaría Villagrasa, M.T. El parque de Ribalta estudio histografico, análisis de vegetación y elementos arquitectónicos.

Traver Tomás, V. (1958). Antigüedades de Castellón de la Plana. Ayuntamiento de Castellón.

Archivos Municipales. Castellón.

Archivo Histórico Municipal. Castellón.

Archivo Diputación Provincial de Castellón.

Fotografías: E. Ballester. 